\title{
Uncertainty as an important determinant in psychological science and practice
}

\author{
Alexander Shorokhov ${ }^{1, *}$ \\ ${ }^{1}$ Don State Technical University, Gagarin Square 1, 344003, Rostov-on-Don, Russia
}

\begin{abstract}
The article reveals the concept of "uncertainty" in the context of culture, science and practice. The notions of uncertainty in different schools and concepts in the field of philosophy and psychology are compared. The hypotheses of the uncertainty phenomenon are explained in detail. The article describes a clinical analysis of five types of subjective attitude to uncertainty, based on the research of scientists: M. K. Mamardashvili, T. E. Sokolova and other experts in the field of subjective attitude to uncertainty. At the end of the article, the data of the scientific interview is given. The article also presents research by modern scientists: Byrne, Peters, Willis, Phan, Worthy (2020), who demonstrated in their research the psychological States of respondents with high uncertainty. The article goes on to describe in more detail the types of research that demonstrate the concepts of acute and moderate stress, tolerance, and other important factors that influence attitudes to uncertainty. The following describes a study aimed at disaster risk reduction, researchers: Schueller, Booth, Fleming, Abad (2020), who developed a disaster risk reduction (DRR) recommendation for stakeholders, which is designed to assess how uncertainty affects the processing of early warning information and subsequent decision-making (for example, an evacuation order), embedded in fictitious geo-graphical, policy and practical conditions. This topic: "Uncertainty as an important determinant in psychological science and practice" is relevant in modern society. The conclusions reveal the content of the data obtained, the analysis of the attitude to uncertainty as a phenomenon of science and practice.
\end{abstract}

\section{Introduction}

Based on the statements of a specialist in historical anthropology, Christoph Wulff, who argued that uncertainty is a defining element of culture, uncertainty is an essential condition for a free, productive and happy human life [1].

As the natural science methodologist Ilya Prigozhin [2] shows, psychologists (Asmolov, 2016) [3] have radically changed the image of science over the course of the twentieth century. Previously, it was believed that science describes stable, deterministic processes. However, the more science learned about nature, the further away from reality this picture turned out to be.

The universal and complete determinism of the material world and human behavior turns out to be an illusion. Moreover, only in non-equilibrium systems described by nonlinear

\footnotetext{
* Corresponding author: solis882008@yandex.ru
} 
equations that have more than one solution can unique, individual events occur, which is the case with any psychotherapist and clinical psychologist. And it is in such non-equilibrium systems, as Prigozhin wrote, that it is possible to expand the scale of this system itself, i.e., to change its attitude to the external world, to the external environment of the system. In other words, only in such systems can true development take place. [4].

The problem of our research is that there is no clear definition of the concept of "uncertainty" in psychology and there is a methodological deficit in the study of this phenomenon. The problem has determined the purpose of our article: to generalize ideas about uncertainty in science and to provide an illustration of its research.

The picture of the world that existed for millennia and deduced all questions of ethics and practical philosophy from the historically established in this culture unshakable value bases, which appear as self-evident, was replaced by a postmodern picture of the world. Postmodernism, which emerged in the twentieth century, philosophically correctly proved that there are no objective grounds for all these value systems, except for the history of their adoption in a certain territory. However, postmodernism did not offer any meaningful alternative to the statement of complete relativity of all value criteria, which results in a violation of the regulation of social life. The only way out of this is to create an internal structure by the individual and to take responsibility for the reasons for their behavior and to develop independently subjective criteria in the absence of any objective external grounds for this [Tulchinsky 2002]. [5].

This is an existential path that is not easy, but has no alternative. People's need for a certain picture of the world is stronger than the need for the truth of this picture, or at least for its plausibility [6]. In the same way, uncertainty is the main, integral characteristic of life in our world. There is a lot of uncertainty in it, and it is absolute, and the segments of certainty are relative. First of all, you need to understand the nature of uncertainty: is it objective or subjective, does it characterize life itself or our consciousness? Kuvakin, Kovaleva [7], analyzing the nature of the unknown, calls it the third reality, along with being and nothing. It seems that such a third ontological state can rather be uncertainty, because the unknown is not an ontological, but an epistemological characteristic, something can be quite definite in its laws and consequences, but for one reason or another unknown to the subject. Moreover, the same phenomenon may be known to one subject and unknown to another, while the characterization of the phenomenon as definite or indefinite does not allow for variations depending on the angle of consideration. Therefore, it is important not to confuse obscurity and uncertainty. [8].

When we talk about uncertainty, we are referring to something objectively indefinite, related to the world order, and not to the limitations of our knowledge (Wakeham, 2015) [9]. When we talk about the objective, we only mean that we are talking about the structure of the world, regardless of how well we understand this device. Here we are talking about a problem that M. Csikszentmihalyi called the "challenge of complexity" of the modern world [10]. It is related to the fact that the world is becoming more and more complex every year, which poses a problem for the individual, how to cope with such complexity, what to do with it. The challenge of complexity can be accepted, or it can be rejected, or it can be avoided. [11].

Accepting the challenge of complexity, a person tries to become more complex himself, continuing to develop all his life. In the modern world, there are many opportunities to develop to old age and death without stopping in this process, but there are also many opportunities NOT to develop, to stop at most after reaching adulthood and forget about further complication and development, to freeze forever in a state of rest. These are two opposite ways of responding to the challenge of the complexity of the modern world, which contains enough opportunities for both strategies. Therefore, personal development after reaching adulthood is an optional process [Leontiev 2013]. [12]. 
The psychological problem of the attitude to uncertainty is largely rooted in the fact that the very concept of uncertainty is difficult to fit into the picture of the world of most people. D. Kahneman in his research, awarded the Nobel prize in Economics [13, 14], and N. Taleb $[15,16]$ in his book "Black Swan", which received worldwide recognition, revealed many mechanisms for simplifying our picture of reality, in particular, reducing uncertainty to certainty, doubtful to certain, according to Heraclitus. "Our mind is an excellent explanatory machine that can find meaning in almost anything, interpret any phenomenon, but is completely unable to accept the idea of unpredictability" [Ibid.: 41]. In another book, he justifies the significance in the modern world of a psychological characteristic, which, for lack of a more appropriate term, he calls "antifragility". The essence of antifragility, its uniqueness "is that it allows us to work with the unknown, do something in conditions when we do not understand what we are doing - and achieve success" [Taleb 2014: 20]. [15, 16].

In the analysis of the problem of uncertainty, various aspects of the attitude to the future come to the future. The future is something that hasn't happened yet, and we can't know for sure if it will, but we constantly expect something, usually with a high degree of subjective certainty. However, there is always a chance that our expectations will be violated, they do not have to be met.

One of the main components of many neurotic problems is overly strict expectations, an excessive degree of subjective certainty that exceeds objective predictability. The future is generally unpredictable, the ability to know the future is an attribute of God, but not of mortal man. Of course, you can predict, expect, and plan something with varying degrees of confidence, but the predictability of the future, including our own actions, is always, to put it mildly, incomplete. This causes us mortals great problems, because we do not want to accept the unpredictability of the future, naturally generates a specific form of psychological protection: a confident understanding of what will happen.

Understanding that the future is not defined creates anxiety, an unpleasant feeling that you do not want to have, and it would be better if it did not exist. However, anxiety is a very important acquisition of a person, it is a tool for interacting with the future. Anxiety shows us that there is something in front of us that we cannot foresee.

If we do not pay attention to the alarm signals, we will fight with the usual, normal anxiety, try to push it out of life, it will still return, only in a more expanded form, and turn into a clinical, pathological anxiety (May, 2015; Leontiev 2003). [17, 18].

By the19th century the natural sciences, which operated with the concept of the material unity of the world, in which everything is determined by definition, took over the baton to assert complete predictability and predestination from religion.

However, in the sciences of the human brain and behavior, the illusion of total certainty has recently given way to the recognition of fundamental uncertainty and the importance of establishing mechanisms for interacting with it [Glimcher 2005]. [19].

Human behavior and mental processes are not completely subject to strict cause-andeffect laws. They contain not only the necessary, causal, but also many things that belong to the category of possible. The thing we think is necessary is the thing without which we cannot be. The thing we think is possible is what may or may not be. The possibility arises in the discontinuity of determination. The concept of possibility is related to the concept of uncertainty, uncertainty means the presence of alternative possibilities. Conversely, an opportunity can only appear where there is incomplete certainty. We talk about the possibility when something in the future is realized through our own actions and cannot be realized in any other way. When something happens or doesn't happen regardless of our own actions, we talk about possibility (see more: [Leontiev 2014]). [20].

The task is to make sure that we ourselves define what is undefined to the end. Selfdetermination means that we ourselves participate in this process, the determinism of the real world. M. K. Mamardashvili metaphorically said that the world has not been created yet to 
the end to our appearance in it, not everything is set in advance, and each person participates in this, creating the world [Mamardashvili 1997]. [21].

Most people are more negative about uncertainty. Clinical analysis of the problem of subjective uncertainty allowed us to describe five types of its experience, of which four are negative [Sokolova 2015: 45-46]. [22].

The first type is an all-consuming negative affect, the content of which is unbearable anxiety.

The second type is also negative emotional state, but lighter phenomenology dominates: ambiguity, ambivalence, ambiguity, unpredictability, inconsistency, confusion, complexity.

The third type is characterized by complete intolerance of uncertainty as a situation of lack of access to internal resources of the "I". The result is extreme dependence on the social environment, conformity, rejection of one's own system of standards, subordination to authority, regime, power, leveling one's own" I".

The fourth type is manic projection, intoxication, transgression and chaos, the absence of any and all borders, any restraining norms and rules of the narcissistic perfectionist type.

The fifth type is an experience tinged with a positive emotional tone: curiosity, searchbased supra-situational activity, fantasy play, generation of new meanings, joy, excitement, pleasure-related research, and insights that lead to a creative and meaningful transformation of the situation of uncertainty.

\section{Materials and methods}

In September 2019, we carried out a pilot study of the understanding and feelings of uncertainty among the students of professional training (total 50 people), the bulk of the experimental group were women aged between 30 and 45 years. Based on the research of professor Elena Sokolova Teodorovna from the Department of neuro - and pathopsychology after M. V. Lomonosov and on the research of Professor E. T. Sokolova [23] (the shock from the collision with the socio-cultural uncertainty: clinical opinion), we conducted research interview and the results are displayed in Table 1.

Table 1. Types of interaction with uncertainty.

\begin{tabular}{|l|c|c|c|c|c|}
\hline \multicolumn{1}{|c|}{ Questions } & \multicolumn{5}{c|}{ Types of interaction with uncertainty } \\
\cline { 2 - 6 } & $\mathbf{1}$ & $\mathbf{2}$ & $\mathbf{3}$ & $\mathbf{4}$ & $\mathbf{5}$ \\
\hline $\begin{array}{l}\text { Is there a state of } \\
\text { uncertainty for you? }\end{array}$ & 2 & 2 & 4 & 3 & 4 \\
\hline $\begin{array}{l}\text { What is uncertainty for } \\
\text { you? }\end{array}$ & 2 & 2 & 4 & 4 & 4 \\
\hline $\begin{array}{l}\text { What do you feel when you } \\
\text { are in a state of } \\
\text { uncertainty? }\end{array}$ & 2 & 3 & 4 & 4 & 6 \\
\hline Total & 6 & 7 & 12 & 11 & 14 \\
\hline$\%$ & 12 & 14 & 24 & 22 & 28 \\
\hline
\end{tabular}

The subjects were asked the following questions:

1) Is there a state of uncertainty (uncertain future) for you?

2) Please tell us what the uncertainty is for you.

3) What do you feel when you are in a state of uncertainty?

\section{Results}

Based on the results of the pilot study, we obtained the following results: both negative and positive perceptions are important in the study. 
The first type - $12 \%$

The second type $-14 \%$

The third type $-24 \%$

The fourth type $-22 \%$

The fifth type $-28 \%$

\section{Discussing results}

Byrne, Peters, Willis, Phan, Worthy (2020) [7] stated in their study that compared to the control state, respondents $(\mathrm{N}=114)$ who were subjected to acute stress chose a highuncertainty option (multiple reward options) that provided a very variable but greater reward than the option that provided a stable, lower reward. The study demonstrated that moderate acute stress is associated with tolerance for variability in outcomes in contexts that depend on learning to maximize reward. Our subjects were also in a moderate stress situation.

Schueller, Booth, Fleming, Abad (2020) developed a disaster risk reduction (DRR) program for stakeholders called RAMSETE III. Its aim is to assess how uncertainty affects the processing of early warning information and subsequent decision-making (such as an evacuation order), embedded in fictitious geographical, political, and practical conditions [24].

We have tried to show that in many ways the acceptance of uncertainty has positive or negative attitudes. This result is combined with the study [25].

We are going to conduct a similar study on the Russian sample.

\section{Conclusions}

Based on our pilot research data, as well as on the materials on the theory and practice of uncertainty, we have come to the conclusion that a positive attitude to uncertainty is possible. This attitude is the most productive for personal development and stress resistance.

\section{References}

1. C. Wulf, Instead of a Preface: uncertainty as a condition of human life. Uncertainty as a challenge. Media. Anthropology. Aesthetics (Russian Christian Humanities Academy, 2013)

2. I. Prigozhin, Question of philosophy 6, 46-52 (1991)

3. A.G. Asmolov, Procedia - Social and Behavioral Sciences 233, 27-34 (2016) https://doi.org/10.1016/j.sbspro.2016.10.122

4. I. Prigogine, I. Stengers, Order from Chaos: Man's New Dialouge with Nature (Bantam Books, 1986) https://books.google.ru/books/about/Order_Out_of_Chaos

5. G.L. Tulchinsky, Posthuman personology. New perspectives of freedom and rationality (Aleteya, St. Petersburg, 2002) https://doi.org/10.12731/2218-7405-2015-2-7

6. A. Tversky, D. Kahneman, Science 211, 453-458 (1981)

7. V.A. Kuvakin, V.P. Kovaleva, Uncertainty (SIC "Regular and chaotic dynamics", Izhevsk, M., 2006)

8. K.A. Byrne, C. Peters, H.C. Willis, D. Phan, D.A. Worthy, Cognition 205, 104448 (2020) https://doi.org/10.1016/j.cognition.2020.104448

9. J. Wakeham, International Encyclopedia of the Social \& Behavioral Sciences (Second Edition) (2015) https://doi.org/10.1016/B978-0-08-097086-8.03175-5 
10. M. Csikszentmihalyi, A Life Worth Living: Contributions to Positive Psychology (Oxford University Press, N.Y., 2006)

11. N.N. Taleb, G. Callahan, Rev Austrian Econ. 21, 361-364 (2008) https://doi.org/10.1007/s11138-008-0051-7

12. D.A. Leontiev, Psychology Questions 3, 67-80 https://doi.org/10.17223/17267080/62/3

13. D. Kahneman, P. Slovik, A. Tversky, Decision-making in uncertainty: Rules and biases (Humanitarian center, Kharkov, 2005)

14. D. Kahneman, J.L. Knetsch, R.H. Thaler, Journal of Political Economy 98(6), 13251348 (1990) DOI: $10.1086 / 261737$

15. N.N. Taleb, The Black Swan. The Impact of the Highly Improbable (Randon House, 2007) https://www.goodreads.com/book/show/242472.The_Black_Swan

16. N.N. Taleb, Antifragility: how to benefit from chaos (Kolibri, M., 2014) https://doi.org/10.1016/j.procs.2014.05.500

17. R. May, The Meaning of Anxiety (W.W. Norton Company, 2015) https://www.goodreads.com/book/show/22253782-the-meaning-of-anxiety

18. D.A. Leontiev, Moscow psychotherapeutic journal 2, $107-119$ (2003) https://doi.org/10.31108/1.2019.1.21.6

19. P.W. Glimcher, Annual Review of Psychology 56, 25-56 (2005) https://doi.org/10.1146/annurev.psych.55.090902.141429

20. D.A. Leontiev, The end of immanence and the prospect of the possible. The place and role of humanism in the future civilization (LENAND, Moscow, 2014)

21. M.K. Mamardashvili, Psychological topology of the path (Russian Christian Humanities Academy, StPb, 1997) DOI 10.33910/herzenpsyconf-2019-2-35

22. E.T. Sokolova, Clinical psychology of I loss (Smysl, M., 2015) DOI: 10.17759/sps. 2017080405

23. K.O. Andreyuk, E.T. Sokolova, National Psychological Journal 12(4), 35-45 (2019) DOI: $10.11621 / \mathrm{npj} .2019 .0404$

24. L. Schueller, L. Booth, K. Fleming, J. Abad, International Journal of Disaster Risk Reduction 51, 101802 (2020) https://doi.org/10.1016/j.ijdrr.2020.101802

25. D.A. Leontiev, Counselling Psychology Quarterly 32(3-4), 548-562 (2019) https://doi.org/10.1080/09515070.2019.1640186 